\title{
On Gorenstein Flat Preenvelopes of Complexes
}

\author{
GANG YAng $(*)$ - Zhongkui LiU $(* *)$ - Li LiAng $(* * *)$
}

ABSTRACT - In this paper we show that if the class $\mathcal{B}$ of $R$-modules is closed under well ordered direct limits, then the class $\mathcal{B}$ is preenveloping in the category of $R$ modules if and only if the class $d w \mathcal{B}$ is preenveloping in the category of $R$ complexes, where $d w \mathcal{B}$ denotes the class of all complexes with all components in $\mathcal{B}$. As an immediate consequence, we get that over commutative and Noetherian rings with dualizing complexes every complex admits a Gorenstein flat preenvelope.

Mathematics Subject Classification (2010). 16E05, $18 \mathrm{G} 35$.

KEYwords. (pre)envelopes, Gorenstein injective modules (complexes), FP-injective modules, Gorenstein flat modules (complexes).

\section{Introduction}

The study of the existence of envelopes and covers started with the introduction of the concepts of injective envelopes ([6]), projective covers ([4]) as well as pure-injective envelopes ([18]). An independent research of Auslander, Reiten and Smal ([2] and [3]) in the finite dimensional case, and Enochs ([7]) for arbitrary modules, has led to a general theory of left and right approximations, or preenvelopes and precovers of modules (see [10] and [15]).

(*) Indirizzo dell'A.: Department of Mathematics, Lanzhou Jiaotong University, Lanzhou 730070, P. R. China.

E-mail: yanggang@mail.lzjtu.cn

(**) Indirizzo dell'A.: Department of Mathematics, Northwest Normal University, Lanzhou 730070, P. R. China.

E-mail: liuzk@nwnu.edu.cn

(***) Indirizzo dell'A.: Department of Mathematics, Lanzhou Jiaotong University, Lanzhou 730070, P. R. China.

E-mail: lliang@mail.lzjtu.cn 
In a natural way, (pre)envelopes and (pre)covers have been studied in more general settings than that of modules. A particular and important example is the study of (pre)envelopes and (pre)covers of complexes of modules (see [1], [13] and [14]). It was shown [14, Theorem 5.2.2] that a ring $R$ is right coherent if and only if every complex of $R$-modules has a flat preenvelope. It was proved [14, Theorem 3.2.9] that over Gorenstein rings every complex has a Gorenstein injective (pre)envelope. In this paper we are inspired to consider the existence of Gorenstein flat preenvelopes. We show that over commutative and Noetherian rings with a dualizing complex every complex admits a Gorenstein flat preenvelope. This complements the work of Enochs, Estrada and Iacob who proved in [8] that over such rings every complex admits a Gorenstein flat (pre)cover. The method we use to show that the class of Gorenstein flat complexes is preenveloping in the category of complexes works in a more general setting, and so the existence of preenvelopes by many important classes of complexes is obtained.

\section{Preliminaries}

Throughout the paper, we assume that all rings have an identity and all modules are unitary. Unless stated otherwise, an $R$-module will be understood to be a left $R$-module. We use $R$-Mod (respectively, Mod- $R$ ) to denote the category of left (respectively, right) $R$-modules.

Definition 2.1. A complex $C$ of $R$-modules is a sequence $\cdots \rightarrow$ $C^{-2} \stackrel{\delta_{C}^{-2}}{\longrightarrow} C^{-1} \stackrel{\delta_{C}^{-1}}{\longrightarrow} C^{0} \stackrel{\delta_{C}^{0}}{\longrightarrow} C^{1} \stackrel{\delta_{C}^{1}}{\longrightarrow} C^{2} \rightarrow \cdots$ of $R$-modules and $R$-homomorphisms such that $\delta_{C}^{m} \delta_{C}^{m-1}=0$ for all $m \in Z$. The $m$ th cycle module of the complex $C$ is defined as $\operatorname{Ker}\left(\delta_{C}^{m}\right)$ and is denoted $Z^{m}(C)$, and the $m$ th boundary module is defined as $\operatorname{Im}\left(\delta_{C}^{m-1}\right)$ and denoted $B^{m}(C)$. A complex $C$ is said to be exact if $Z^{m}(C)=B^{m}(C)$ for all $m \in Z$.

Definition 2.2 ([10]). A module $M$ is called Gorenstein injective, if there exists an exact complex $\cdots \rightarrow I^{-2} \rightarrow I^{-1} \rightarrow I^{0} \rightarrow I^{1} \rightarrow I^{2} \rightarrow \cdots$ with all $I^{i}$ injective such that $M=\operatorname{Ker}\left(I^{0} \rightarrow I^{1}\right)$ and the sequence remains exact whenever $\operatorname{Hom}_{R}(J,-)$ is applied for any injective module $J$.

Definition 2.3 ([10]). A module $N$ is called Gorenstein flat, if there exists an exact complex $\cdots \rightarrow F^{-2} \rightarrow F^{-1} \rightarrow F^{0} \rightarrow F^{1} \rightarrow F^{2} \rightarrow \cdots$ with all $F^{i}$ flat such that $N=\operatorname{Ker}\left(F^{0} \rightarrow F^{1}\right)$ and the sequence remains exact whenever $J \otimes_{R}$ - is applied for any injective right $R$-module $J$. 
The Gorenstein injective and flat complexes are defined in a similar manner, but using resolutions of complexes.

Given complexes $X$ and $Y$, we let $\mathcal{H o m}(X, Y)$ denote the usual complex associated complexes $X$ and $Y$, i.e., the complex with $\mathcal{H o m}(X, Y)^{n}=$ $\prod_{i \in Z} \operatorname{Hom}\left(X^{i}, Y^{n+i}\right)$ and with differential given by $\delta^{n}\left(\left(f^{i}\right)_{i \in Z}\right)=\left(\delta_{Y}^{n+i} f^{i}-\right.$ $\left.(-1)^{n} f^{i+1} \delta_{X}^{i}\right)_{i \in Z}$ for $f=\left(f^{i}\right)_{i \in Z}$ in $\mathcal{H o m}(X, Y)^{n}$. A morphism of complexes $f: X \rightarrow Y$ is defined to be a family $\left(f^{i}\right)_{i \in Z}$ of homomorphisms of $R$-modules $f^{i}: X^{i} \rightarrow Y^{i}$ such that $\delta_{Y}^{i} f^{i}=f^{i+1} \delta_{X}^{i}$ for each $i \in Z$. We use $\operatorname{Hom}(X, Y)$ to present the group of all morphisms from $X$ to $Y$. We let $C(R$-Mod) (respectively, $C(\operatorname{Mod}-R)$ ) be the category of complexes of left (respectively, right) $R$-modules. Unless stated otherwise, an $R$-complex will be understood to be a complex of left $R$-modules.

We also recall that a complex $I$ is injective if the functor $\operatorname{Hom}(-, I)$ is exact. Equivalently, a complex $I$ injective is if and only if it is exact and $Z^{i}(I)$ is an injective module for each $i \in Z$. For example, if $N$ is an injective module then $\cdots \rightarrow 0 \rightarrow N \stackrel{I d}{\longrightarrow} N \rightarrow 0 \rightarrow \cdots$ is injective. In fact, any injective complex is uniquely up to isomorphism the direct sum of such complexes. The dual notion is that of projective complex. A complex $P$ is projective if the functor $\operatorname{Hom}(P,-)$ is exact. Equivalently, $P$ is projective if and only if $P$ is exact and $Z^{i}(P)$ is a projective module for each $i \in Z$. For example, if $M$ is a projective module then $\cdots \rightarrow 0 \rightarrow M \stackrel{I d}{\longrightarrow} M \rightarrow 0 \rightarrow \cdots$ is projective. In fact, any projective complex is uniquely up to isomorphism the direct sum of such complexes. Thus the category of complexes of modules has enough projectives.

Definition 2.4 ([14]). A complex $H$ is called Gorenstein injective, if there exists an exact sequence of complexes $\cdots \rightarrow I^{-2} \rightarrow I^{-1} \rightarrow I^{0} \rightarrow$ $I^{1} \rightarrow I^{2} \rightarrow \cdots$ with all $I^{i}$ injective such that $H=\operatorname{Ker}\left(I^{0} \rightarrow I^{1}\right)$ and the sequence remains exact whenever $\operatorname{Hom}(J,-)$ is applied for any injective complex $J$.

REMARK 2.5. It was shown by Liu and Zhang [17, Theorem 8] that a complex $H$ is Gorenstein injective if and only if each term $H^{i}$ is a Gorenstein injective module for all $i \in Z$ when $R$ is left Noetherian.

The definition of Gorenstein flat complexes is introduced in terms of tensor product of complexes.

Recall from [14] that if $X$ is a complex of right $R$-modules and $Y$ is a complex of left $R$-modules, then the usual tensor product $X \otimes Y$ is defined 
by $(X \otimes Y)^{n}=\bigoplus_{i+j=n} X^{i} \otimes_{R} Y^{j}$ in degree $n$, and the differential $\delta^{n}$ is defined on the generators by $\delta_{X}^{i}(x) \otimes y+(-1)^{i} x \otimes \delta_{Y}^{j}(y)$ for $x \in X^{i}, y \in Y^{j}$. Let $X \otimes Y$ denote the complex $\frac{(X \otimes Y)}{B(X \otimes Y)}$ with the maps

$$
\frac{(X \otimes Y)^{n}}{B^{n}(X \otimes Y)} \rightarrow \frac{(X \otimes Y)^{n+1}}{B^{n+1}(X \otimes Y)}, x \otimes y \mapsto \delta_{X}(x) \otimes y,
$$

where $x \otimes y$ is used to denote the coset in $\frac{(X \otimes Y)^{n}}{B^{n}(X \otimes Y)^{\prime}}$. Then we note that the functor $-\otimes-$ is right exact in the category of complexes and the category of complexes has enough projectives, thus we can construct left derived functors which we denote by Tor $_{i}$.

Recall from [14] that a complex $F$ is flat if the functor $-\otimes F$ is exact. By [14, Theorem 4.1.3], a complex $F$ is flat if and only if $F$ is exact and $Z^{i}(F)$ is a flat module for each $i \in Z$. It is clear that any projective complex is flat.

Definition 2.6 ([14]). A complex $G$ is called Gorenstein flat if there exists an exact sequence of complexes $\cdots \rightarrow F^{-2} \rightarrow F^{-1} \rightarrow F^{0}$ $\rightarrow F^{1} \rightarrow F^{2} \rightarrow \cdots$ with all $F^{i}$ flat such that $G=\operatorname{Ker}\left(F^{0} \rightarrow F^{1}\right)$ and the sequence remains exact whenever $E \otimes-$ is applied for any injective complex $E$ of right $R$-modules.

We also recall the definitions of precovers and preenvelopes as well as right and left resolutions in an abelian category $\mathcal{A}$.

Definition 2.7. Let $\Omega$ be a class of objects of $\mathcal{A}$, and $M$ is an object of $\mathcal{A}$. We say that a morphism $f: M \rightarrow Q$ is an $\Omega$-preenvelope if $Q \in \Omega$ and the sequence $\operatorname{Hom}\left(Q, Q^{\prime}\right) \rightarrow \operatorname{Hom}\left(M, Q^{\prime}\right) \rightarrow 0$ is exact for any $Q^{\prime} \in \Omega$. If moreover, $g \circ f=f$ implies that $g$ is an automorphism whenever $g \in \operatorname{End}(Q)$, then $f$ is called an $\Omega$-envelope. $\Omega$-precovers and $\Omega$-covers are defined dually. We say that the class $\Omega$ is preenveloping (respectively, enveloping) if every object of $\mathcal{A}$ has an $\Omega$-preenvelope (respectively, $\Omega$ envelope).

Definition 2.8. A right $\Omega$-resolution of $M$ is a complex $0 \rightarrow M \rightarrow$ $Q^{0} \rightarrow Q^{1} \rightarrow \cdots$ with each $Q^{i} \in \Omega$ and which remains exact whenever $\operatorname{Hom}\left(-, Q^{\prime}\right)$ is applied for any $Q^{\prime} \in \Omega$. We note that $0 \rightarrow M \rightarrow$ $Q^{0} \rightarrow Q^{1} \rightarrow \cdots$ is a right $\Omega$-resolution of $M$ if and only if $M \rightarrow Q^{0}$ and each $\operatorname{Coker}\left(Q^{i-2} \rightarrow Q^{i-1}\right) \rightarrow Q^{i}$ for $i \geq 1$ are $\Omega$-preenvelopes (with $Q^{-1}=M$ ). 
The left $\Omega$-resolutions are defined dually. Note that if $\Omega$ contains all injective (respectively, projective) objects then any right (respectively, left) $\Omega$-resolution is an exact complex.

We note that there is no loss of generality in what follows in assuming that if $\mathcal{B}$ is a class of $R$-modules and $M, N$ are $R$-modules such that $M \cong N$ and $M \in \mathcal{B}$, then $N \in \mathcal{B}$. Hence we will always assume that the class $\mathcal{B}$ is closed under isomorphisms and contains 0 . Let $\mathcal{B}$ be a class of $R$-modules, we say a complex $D$ is a $d w \mathcal{B}$ complex if each term $D^{i}$ is in $\mathcal{B}$ for $i \in Z$ (the " $d w$ " is meant to be thought of as "degreewise").

\section{Gorenstein flat complexes over coherent rings}

The aim of this section is to give some characterizations of Gorenstein flat complexes over coherent rings. So throughout this section $R$ will denote a right coherent ring.

Since over a right coherent ring every module has a flat preenvelope by [10, Proposition 3.3], then every module has a right flat resolution. Thus one can define right derived functors of $-\otimes_{R}$ - by using (right) injective resolutions and right flat resolutions in the first and second variables respectively (see [10]). These new derived functors are denoted by $\operatorname{Tor}_{R}^{n}$, and such functors can be used to characterize Gorenstein flat modules (see [11, Proposition 2.1]). Similarly since over a right coherent ring every complex has a flat preenvelope by [14, Theorem 5.2.2], then every complex has a right flat resolution. Thus we can define right derived functors of $-\otimes-$ in the category of complexes by using (right) injective resolutions and right flat resolutions in the first and second variables respectively. These new derived functors are denoted by $\operatorname{Tor}^{n}$. We also note that there exists a natural morphism $X \otimes Y \rightarrow \operatorname{Tor}^{0}(X, Y)$.

Given an $R$-module $M$, we denote by $\bar{M}$ the complex $\cdots \rightarrow 0 \rightarrow$ $M \stackrel{I d}{\longrightarrow} M \rightarrow 0 \rightarrow \cdots$ with all components 0 except $M$ in the degrees -1 and 0 . Given a complex $C$ and an integer $m$, we let $C[m]$ denote the complex such that $C[m]^{n}=C^{m+n}$ and whose differentials are $(-1)^{m} \delta^{m+n}$. We first give the following useful lemma.

Lemma 3.1. Let $C$ be a complex, $M$ a right $R$-module and let $m$ be an integer. Then the following statements hold.

(1) $\left[\operatorname{Tor}^{n}(\bar{M}[m], C)\right]^{i} \cong \operatorname{Tor}_{R}^{n}\left(M, C^{i+m}\right)$ for any $i \in Z$ and any $n \geq 0$.

(2) $\left[\operatorname{Tor}_{n}(\bar{M}[m], C)\right]^{i} \cong \operatorname{Tor}_{n}^{R}\left(M, C^{i+m}\right)$ for any $i \in Z$ and any $n \geq 0$. 
Proof. (1). Let $0 \rightarrow M \rightarrow I^{0} \rightarrow I^{1} \rightarrow I^{2} \rightarrow \cdots$ be an injective resolution of the module $M$. Then $0 \rightarrow \bar{M}[m] \rightarrow \overline{I^{0}}[m] \rightarrow \overline{I^{1}}[m] \rightarrow \overline{I^{2}}[m] \rightarrow \cdots$ is an injective resolution of the complex $\bar{M}[m]$, and so we have the following commutative diagram

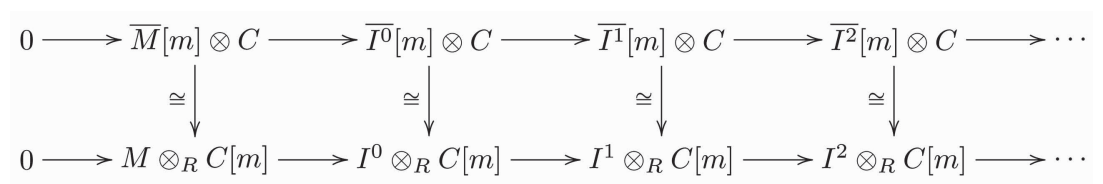

where the vertical isomorphisms follow from [14, Proposition 4.2.1(4)]. Now it is easy to see that $\left[\operatorname{Tor}^{n}(\bar{M}[m], C)\right]^{i} \cong \operatorname{Tor}_{R}^{n}\left(M, C^{i+m}\right)$ for any $i \in Z_{s}$ and any $n \geq 0$.

(2). The proof is similar to that of (1).

Note that $\operatorname{Hom}(X, Y)=Z^{0}(\mathcal{H o m}(X, Y))$. If we let $\operatorname{Hom}(X, Y)=$ $Z(\mathcal{H o m}(X, Y))$ then we see that $\operatorname{Hom}(X, Y)$ can be made into a complex with $\underline{\operatorname{Hom}}(X, Y)^{m}$ the abelian group of morphisms from $X$ to $Y[m]$ and with a differential given by: $f \in \underline{\operatorname{Hom}}(X, Y)^{m}$, then $\delta^{m}(f): X \rightarrow Y[m+1]$ with $\delta^{m}(f)^{n}=(-1)^{m} \delta_{Y}^{m+n} f^{n}, \forall n \in Z$. For a complex $C$, we let $C^{+}$denote the complex $\underline{\operatorname{Hom}}(C, \overline{\mathrm{Q} / \mathrm{Z}})$, that is, $C^{+}=\operatorname{Hom}_{R}(C, \mathrm{Q} / \mathrm{Z})$.

In the following we give a characterization of Gorenstein flat complexes over right coherent rings by using the new derived functors Tor ${ }^{n}$.

Proposition 3.2. Let $G$ be a complex. Then the following conditions are equivalent.

(1) $G$ is Gorenstein flat.

(2) $\operatorname{Tor}_{n}(E, G)=0 \forall n \geq 1$ for every injective complex $E$ of right $R$-modules, $\operatorname{Tor}^{n}(\bar{R}, G)=0 \quad \forall n \geq 1$ and $G \rightarrow \operatorname{Tor}^{0}(\bar{R}, G)$ is an isomorphism.

(3) $G^{i}$ are Gorenstein flat modules for all $i \in \mathrm{Z}$.

(4) $\operatorname{Tor}_{n}(E, G)=0 \forall n \geq 1$ for every injective complex $E$ of right $R$ modules, $\operatorname{Tor}^{n}(P, G)=0 \forall n \geq 1$ and $P \otimes G \rightarrow \operatorname{Tor}^{0}(P, G)$ is an isomorphism for every projective complex $P$.

Proof. $\quad(1) \Rightarrow(2)$. It is clear that $\operatorname{Tor}_{n}(E, G)=0 \forall n \geq 1$ for every injective complex $E$ of right $R$-modules. Furthermore since $R$ is right coherent then there exists an exact sequence $0 \rightarrow G \rightarrow F^{0} \rightarrow F^{1} \rightarrow \cdots$ that can be used to compute $\operatorname{Tor}^{n}(\bar{R}, G)=0, \forall n \geq 1$. Finally $G=$ $\operatorname{Ker}\left(F^{0} \rightarrow F^{1}\right) \cong \operatorname{Ker}\left(\bar{R} \otimes F^{0} \rightarrow \bar{R} \otimes F^{1}\right)=\operatorname{Tor}^{0}(\bar{R}, G)$. 
$(2) \Rightarrow(3)$. It follows from Lemma 3.1 and [11, Proposition 2.1].

$(3) \Rightarrow(4)$. Since the derived functors Tor $_{n}$ and Tor $^{n}$ of the tensor product preserve arbitrary direct sums, every projective module is a direct summand of a free $R$-module and every projective complex $P$ is a direct sum of some projective complexes $\bar{K}[i]$, then the result follows from Lemma 3.1 and [11, Proposition 2.1].

(4) $\Rightarrow(1)$. Let $0 \rightarrow G \rightarrow F^{0} \rightarrow F^{1} \rightarrow \cdots$ be a right flat resolution. Then by the fact that $\operatorname{Tor}_{n}(\bar{R}, G)=0 \forall n \geq 1$ we get that $F^{0} \rightarrow F^{1} \rightarrow \cdots$ is exact. But since $G \cong \bar{R} \otimes G \rightarrow \operatorname{Tor}^{0}(\bar{R}, G)=\operatorname{Ker}\left(\bar{R} \otimes F^{0} \rightarrow \bar{R} \otimes F^{1}\right)$ is an isomorphism, we get that $0 \rightarrow G \rightarrow F^{0} \rightarrow F^{1} \rightarrow \cdots$ is exact. Let $E$ be an injective complex of right $R$-modules. In order to show that $0 \rightarrow E \otimes G \rightarrow E \otimes F^{0} \rightarrow E \otimes F^{1} \rightarrow \cdots$ is exact, it is enough to show that $\cdots \rightarrow\left(E \otimes F^{1}\right)^{+} \rightarrow\left(E \otimes F^{0}\right)^{+} \rightarrow(E \otimes G)^{+} \rightarrow 0$ is exact. But this follows from isomorphisms $(E \otimes C)^{+} \cong \underline{\operatorname{Hom}}\left(C, E^{+}\right)$for any complex $C$, and the exactness of the sequence $\cdots \rightarrow \operatorname{Hom}\left(F^{1}, E^{+}\right) \rightarrow \underline{\operatorname{Hom}}\left(F^{0}, E^{+}\right) \rightarrow$ $\underline{\operatorname{Hom}}\left(G, E^{+}\right) \rightarrow 0$ because $E^{+}$is flat. On the other hand $\operatorname{Tor}_{n}(E, G)=0$ $\forall n \geq 1$ implies that any (left) flat resolution $\cdots \rightarrow F_{1} \rightarrow F_{0} \rightarrow G \rightarrow 0$ remains exact whenever the functor $E \otimes-$ is applied for any injective complex $E$ of right $R$-modules.

REMARK 3.3. The equivalence of statements (1) and (3) of Proposition 3.2 was shown over more general rings by Yang and Liu [21, Theorem 3.11].

Since a left $R$-module $M$ is Gorenstein flat if and only if the character module $M^{+}$is Gorenstein injective as a right $R$-module by [16, Theorem 3.6], the following result follows directly from Remark 2.5 and Proposition 3.2.

COROLLARY 3.4. Let $R$ be a right Noetherian ring. Then a complex $G$ is Gorenstein flat in $C\left(R\right.$-Mod) if and only if $G^{+}$is Gorenstein injective in $C(\operatorname{Mod}-R)$.

\section{The existence of Gorenstein flat preenvelopes}

In this section we will prove that over commutative and Noetherian rings with a dualizing complex every complex has a Gorenstein flat preenvelope. To obtain this result, we develop a general approach. More precisely, for a given class $\mathcal{B}$ of $R$-modules we will show that if the class $\mathcal{B}$ of $R$-modules is closed under direct limits, then the class $\mathcal{B}$ is preenveloping in $R$-Mod if and only if the class $d w \mathcal{B}$ of complexes is preenveloping in 
$C(R$-Mod), where $d w \mathcal{B}$ denotes the class of complexes of all components in $\mathcal{B}$. We first start with the following lemma.

Lemma 4.1. If the class of $R$-modules $\mathcal{B}$ is enveloping in $R$-Mod then every left bounded complex has a dwB-envelope. Furthermore, if the class of $R$-modules $\mathcal{B}$ is preenveloping in $R$-Mod then every left bounded complex has a dwB-preenvelope which is also left bounded.

Proof. We assume without loss of generality that $C=: 0 \rightarrow$ $C^{0} \stackrel{\delta_{C}^{0}}{\longrightarrow} C^{1} \stackrel{\delta_{C}^{1}}{\longrightarrow} C^{2} \rightarrow \cdots$. Using an idea dual to the one used in [14, Theorem 3.3.10], we are going to construct a complex $D \in d w \mathcal{B}$ and a morphism of complexes $f: C \rightarrow D$ which is a $d w \mathcal{B}$-envelope of $C$ in the following way.

Firstly, for $n<0$, let $D^{n}=0$ and $f^{n}=0$. Let $f^{0}: C^{0} \rightarrow D^{0}$ be a $\mathcal{B}$-envelope of $C^{0}$. Now for $n \geq 0$, we proceed inductively. Suppose that we have constructed:

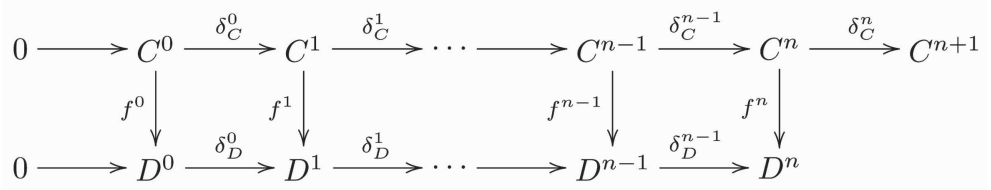

We consider the push-out diagram of $\delta_{C}^{n}: C^{n} \rightarrow C^{n+1}$ and $\pi^{n} f^{n}: C^{n} \rightarrow$ $\operatorname{Coker}\left(\delta_{D}^{n-1}\right)$,

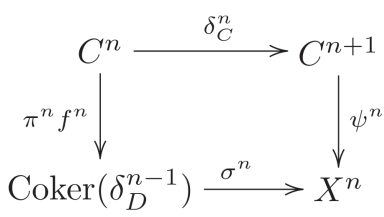

where $\pi^{n}: D^{n} \rightarrow \operatorname{Coker}\left(\delta_{D}^{n-1}\right)$ is the natural epimorphism.

Then we take a $\mathcal{B}$-envelope $\tau^{n}: X^{n} \rightarrow D^{n+1}$ of the module $X^{n}$ and define $\delta_{D}^{n}: D^{n} \rightarrow D^{n+1}$ as the composition $\delta_{D}^{n}=\tau^{n} \sigma^{n} \pi^{n}$ and $f^{n+1}: C^{n+1} \rightarrow D^{n+1}$ as the composition $f^{n+1}=\tau^{n} \psi^{n}$. It is easy to see that $\delta_{D}^{n} \delta_{D}^{n-1}=0\left(\delta_{D}^{n-1}=0\right.$ if $n=0)$ and thus this construction produces a complex $D \in d w \mathcal{B}$ and a morphism of complexes $f: C \rightarrow D$.

Secondly, we will show that the above morphism of complexes $f: C \rightarrow D$ is a $d w \mathcal{B}$-preenvelope of the complex $C$. Let $A \in d w \mathcal{B}$ and $\varphi: C \rightarrow A$ be a morphism of complexes. We are going to construct a morphism of complexes $\gamma: D \rightarrow A$ satisfying $\varphi=\gamma f$. For $n<0$, we take $\gamma^{n}=0$. For $n=0$, since $f^{0}: C^{0} \rightarrow D^{0}$ is a $\mathcal{B}$-envelope of $C^{0}$, there exists 
$\gamma^{0}: D^{0} \rightarrow A^{0}$ such that $\gamma^{0} f^{0}=\varphi^{0}$. By induction, suppose $\gamma^{n}: D^{n} \rightarrow A^{n}$ is defined such that $\gamma^{n} f^{n}=\varphi^{n}$ and $\gamma^{n} \delta_{D}^{n-1}=\delta_{A}^{n-1} \gamma^{n-1}$. We notice that $\operatorname{Ker}\left(\pi^{n}\right) \subset \operatorname{Ker}\left(\delta_{A}^{n} \gamma^{n}\right)$. Since $\pi^{n}: D^{n} \rightarrow \operatorname{Coker}\left(\delta_{D}^{n-1}\right)$ is epimorphic, by the factor lemma, there exists a morphism $g^{n}: \operatorname{Coker}\left(\delta_{D}^{n-1}\right) \rightarrow A^{n+1}$ such that the following diagram is commutative:

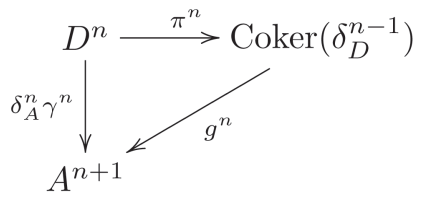

i.e., we have $g^{n} \pi^{n}=\delta_{A}^{n} \gamma^{n}$. Hence $g^{n} \pi^{n} f^{n}=\delta_{A}^{n} \gamma^{n} f^{n}=\delta_{A}^{n} \varphi^{n}=\varphi^{n+1} \delta_{C}^{n}$. This yields the induced commutative diagram by the following push-out diagram:

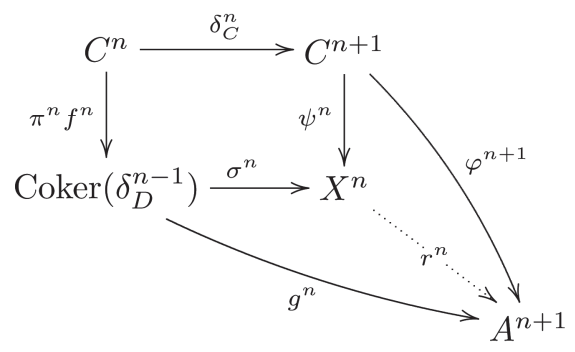

Since $\tau^{n}: X^{n} \rightarrow D^{n+1}$ is a $\mathcal{B}$-envelope of $X^{n}$ and $A^{n+1}$ is in $\mathcal{B}$, there exists $\gamma^{n+1}: D^{n+1} \rightarrow A^{n+1}$ such that $\gamma^{n+1} \tau^{n}=r^{n}$. Therefore, we get from the commutative diagram (2) that $\gamma^{n+1} f^{n+1}=\gamma^{n+1} \tau^{n} \psi^{n}=r^{n} \psi^{n}=\varphi^{n+1}$. By above construction, it is not hard to check that $\gamma^{n+1} \delta_{D}^{n}=\delta_{A}^{n} \gamma^{n}$. So it is easy to see that in this way we can get a morphism of complexes $\gamma: D \rightarrow A$ satisfying $\gamma f=\varphi$, thus we proved.

In the last, let $\gamma: D \rightarrow D$ be a morphism of complexes such that $\gamma f=f$. For $n<0$, we have that $\gamma^{n}$ is the zero morphism. For $n=0$, we have that $\gamma^{0}$ is an automorphism since $f^{0}: C^{0} \rightarrow D^{0}$ is a $\mathcal{B}$-envelope of $C^{0}$. For $n \geq 0$, we proceed inductively. Suppose that $\gamma^{0}, \gamma^{1}, \cdots, \gamma^{n}$ are automorphisms, we will show that $\gamma^{n+1}: D^{n+1} \rightarrow D^{n+1}$ is also an automorphism. By the factor lemma, there exists a morphism $\overline{\gamma^{n}}: \operatorname{Coker}\left(\delta_{D}^{n-1}\right) \rightarrow \operatorname{Coker}\left(\delta_{D}^{n-1}\right)$ such that the following diagram commutes:

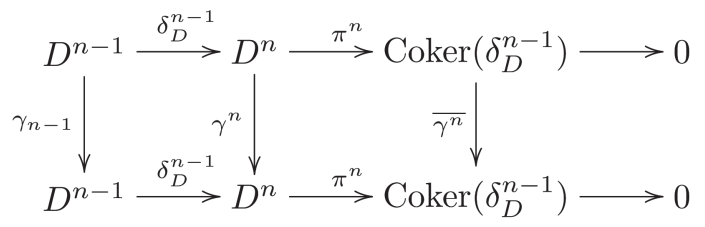


By the five lemma, we get that $\overline{\gamma^{n}}: \operatorname{Coker}\left(\delta_{D}^{n-1}\right) \rightarrow \operatorname{Coker}\left(\delta_{D}^{n-1}\right)$ is an isomorphism. Combining the commutative diagrams (1) and (3), we have $\psi^{n} \delta_{C}^{n}=$ $\sigma^{n} \pi^{n} f^{n}=\sigma^{n} \pi^{n}\left(\gamma^{n} f^{n}\right)=\sigma^{n}\left(\pi^{n} \gamma^{n}\right) f^{n}=\sigma^{n}\left(\overline{\gamma^{n}} \pi^{n}\right) f^{n}$. Hence, the push-out diagram

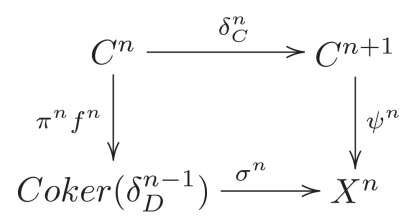

maps into itself by an automorphism. This implies that there exists an automorphism $\widehat{\gamma^{n}}: X^{n} \rightarrow X^{n}$ such that $\widehat{\gamma}^{n} \psi^{n}=\psi^{n}$ and the following diagram commutes on each surface

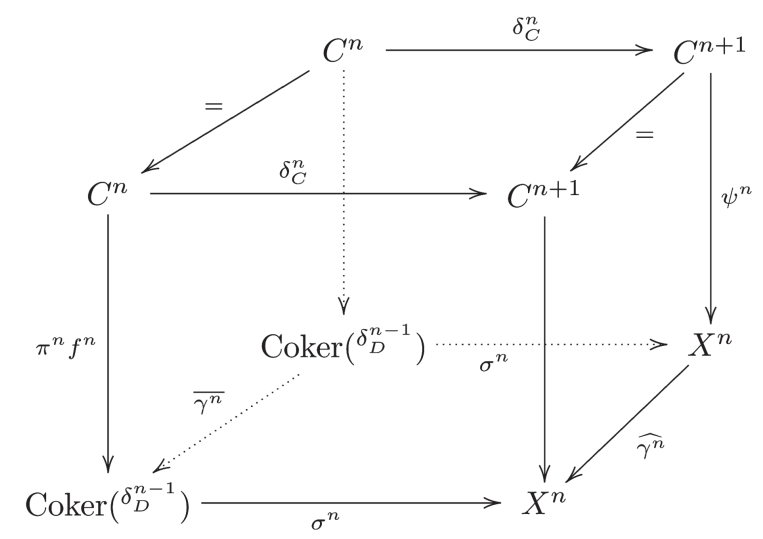

Since

$$
\begin{aligned}
f^{n+1} \delta_{C}^{n} & =\delta_{D}^{n} f^{n}=\tau^{n} \sigma^{n} \pi^{n} f^{n}=\tau^{n} \sigma^{n} \pi^{n}\left(\gamma^{n} f^{n}\right)=\tau^{n} \sigma^{n}\left(\pi^{n} \gamma^{n}\right) f^{n} \\
& =\tau^{n} \sigma^{n}\left(\overline{\gamma^{n}} \pi^{n}\right) f^{n}=\tau^{n}\left(\sigma^{n} \overline{\gamma^{n}}\right) \pi^{n} f^{n}=\tau^{n}\left(\widehat{\gamma^{n}} \sigma^{n}\right) \pi^{n} f^{n} \\
& =\left(\tau^{n}{\widehat{\gamma^{n}}}^{n}\right)\left(\pi^{n} f^{n}\right),
\end{aligned}
$$

there exists an unique morphism $\omega: X^{n} \rightarrow D^{n+1}$ such that the following diagram is commutative

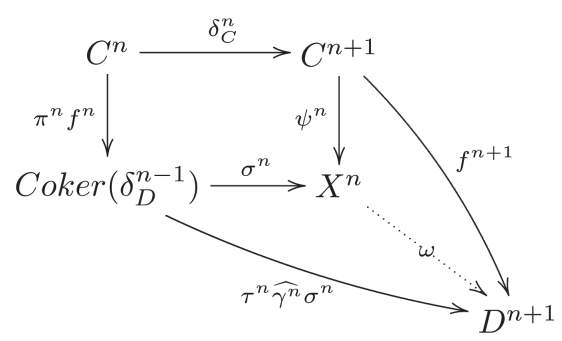


By standard computation, we can substitute $\omega$ with either $\tau^{n} \widehat{\gamma^{n}}$ or $\gamma^{n+1} \tau^{n}$, so they are identical, i.e., $\tau^{n} \widehat{\gamma^{n}}=\gamma^{n+1} \tau^{n}$, where $\tau^{n}: X^{n} \rightarrow D^{n+1}$ is a $\mathcal{B}$-envelope of $X^{n}$. We get that $\gamma^{n+1}: D^{n+1} \rightarrow D^{n+1}$ is an automorphism. By induction, we get that $\gamma: D \rightarrow D$ is an automorphism. This shows that every left bounded complex has a $d w \mathcal{B}$-envelope.

To complete the proof it only remains to argue that if the class of $R$ modules $\mathcal{B}$ is preenveloping in $R$-Mod then every left bounded complex has a $d w \mathcal{B}$-preenvelope which is also left bounded. But this is obvious from the proof above.

The following theorem gives our main result in this section, which presents a nice relation of preenvelopes between $R$-modules and unbounded complexes of $R$-modules.

THEOREM 4.2. Let $\mathcal{B}$ be a class of $R$-modules which is closed under well ordered direct limits. Then $\mathcal{B}$ is preenveloping in $R$-Mod if and only if the class $d w \mathcal{B}$ is preenveloping in $C(R-M o d)$.

Proof. For the necessity, we need only to show that every complex of $R$-modules has a $d w \mathcal{B}$-preenvelope. Let

$$
A=: \cdots \rightarrow A_{2} \stackrel{\partial_{2}^{A}}{\longrightarrow} A_{1} \stackrel{\partial_{1}^{A}}{\longrightarrow} A_{0} \stackrel{\partial_{0}^{4}}{\longrightarrow} A_{-1} \rightarrow \cdots
$$

and we write

$$
A(n)=: \quad 0 \rightarrow A_{n} \stackrel{\partial_{n}^{A}}{\longrightarrow} A_{n-1} \stackrel{\partial_{n-1}^{A}}{\longrightarrow} A_{n-2} \stackrel{\partial_{n-2}^{4}}{\longrightarrow} \cdots
$$

Then we get that $\left((A(n)),\left(\rho_{m n}\right)\right)_{n \geq 0}$ is a well ordered direct system in $C(R$-Mod $)$ and $\lim A(n)=A$, where $\rho_{m n}: A(m) \rightarrow A(n)$ is a natural injection for any $m \leq n$.

By Lemma 4.1, there exists a $d w \mathcal{B}$-preenvelope $\eta_{0}: A(0) \rightarrow D(0)$ of $A(0)$ with $D(0)$ left bounded. Then we consider the push-out diagram of morphisms $\eta_{0}: A(0) \rightarrow D(0)$ and $\rho_{01}: A(0) \rightarrow A(1)$

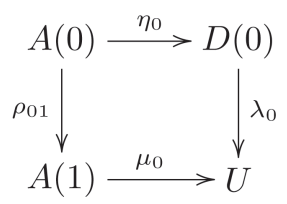

Clearly, $U$ is left bounded Since the others of above diagram are so. Again using Lemma 4.1, we have a $d w \mathcal{B}$-preenvelope $v: U \rightarrow D(1)$ of $U$ with $D(1)$ 
left bounded. In the following we will show that the composition $v \mu_{0}: A(1) \rightarrow D(1)$ is a $d w \mathcal{B}$-preenvelope of $A(1)$. Suppose that $\alpha: A(1) \rightarrow B$ is any morphism with $B$ a $d w \mathcal{B}$ complex. Then there exists a morphism $\widetilde{\alpha}: D(0) \rightarrow B$ such that $\alpha \rho_{01}=\widetilde{\alpha} \eta_{0}$ since $\eta_{0}: A(0) \rightarrow D(0)$ is a $d w \mathcal{B}$-preenvelope of $A(0)$, and so we have a morphism $\widehat{\alpha}: U \rightarrow B$ such that the completed diagram

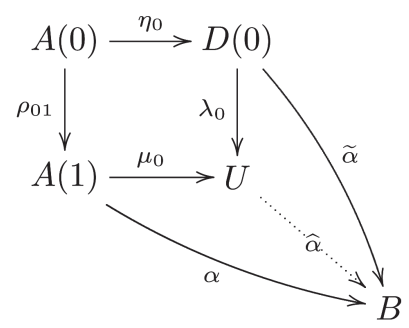

commutes. Since $v: U \rightarrow D(1)$ is a $d w \mathcal{B}$-preenvelope of $U$, there exists a morphism $\beta: D(1) \rightarrow B$ such that $\widehat{\alpha}=\beta v$, and so $\alpha=\widehat{\alpha} \mu_{0}=\beta v \mu_{0}$. This proves that $v \mu_{0}: A(1) \rightarrow D(1)$ is a $d w \mathcal{B}$-preenvelope of $A(1)$. Therefore we get, by the construction above, a commutative diagram

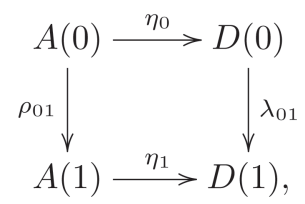

where $\lambda_{01}=v \lambda_{0}$ and $\eta_{1}=v \mu_{0}$, and it has the property that if

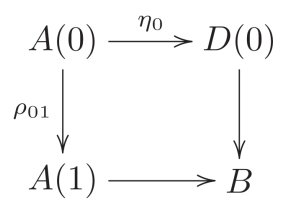

is commutative with $B$ in $d w \mathcal{B}$ then there exists a morphism of complexes $D(1) \rightarrow B$ such that the completed diagram

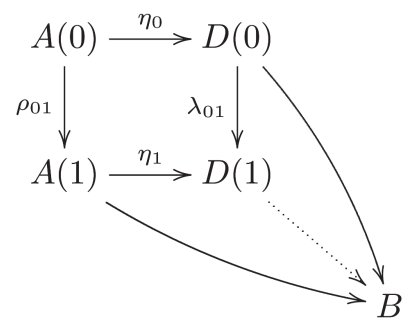


commutes. If we continue this process, we get a commutative diagram

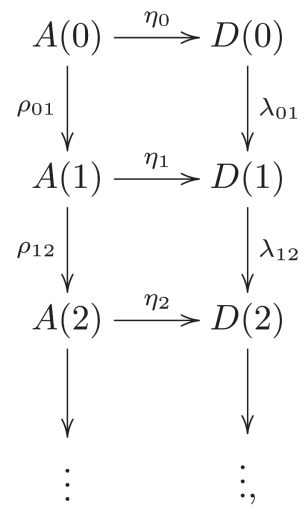

where $\eta_{n}: A(n) \rightarrow D(n)$ is a $d w \mathcal{B}$-preenvelope of $A(n)$ for each $n \geq 0$ and each diagram

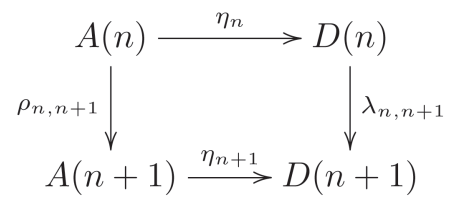

has the preceding property. Clearly, $\left((D(n)),\left(\lambda_{m n}\right)\right)_{n \geq 0}$ forms a well ordered direct system in $C(R$-Mod).

Note that $d w \mathcal{B}$ is closed under well ordered direct limits and we have $\lim D(n)$ in $d w \mathcal{B}$. We will see in the following that the morphism $\eta: \lim A(n) \rightarrow \lim D(n), \eta=\lim \eta_{n}$, is a dwB-preenvelope of $\lim A(n)=A$. In fact, if $f: \vec{A} \rightarrow X$ is a morphism with $X \in d w \mathcal{B}$, then there exists $\chi_{0}: D(0) \rightarrow X$ such that the following diagram

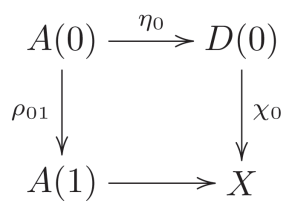

commutes since $\eta_{0}: A(0) \rightarrow D(0)$ is a $d w \mathcal{B}$-preenvelope of $A(0)$, where $A(1) \rightarrow X$ is the composition of morphisms of the natural injection $A(1) \rightarrow A$ and $f: A \rightarrow X$. Thus, by construction above, there is a morphism $\chi_{1}: D(1) \rightarrow X$ such that the completed diagram 


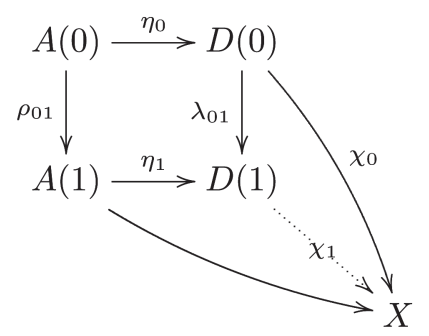

commutes. By using this procedure continuously, we get that the following diagram

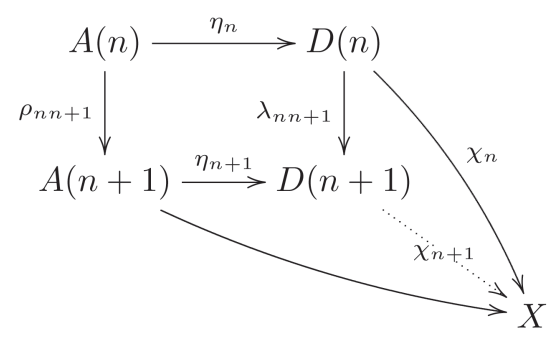

is commutative for any $n \geq 0$, now it is easy to see that there exists a morphism $\chi: \lim D(n) \rightarrow X$ such that $f=\chi \eta$. Thus $\eta: \lim A(n) \rightarrow \lim D(n)$ is a $d w \mathcal{B}$-preenvelope of $\underset{\lim }{\longrightarrow} A(n)=A$.

For the sufficiency, let $M$ be an $R$-module and consider $f: \bar{M} \rightarrow D$ a $d w \mathcal{B}$-preenvelope of $\bar{M}$ in $C(R$-Mod). Then it is easy to see that $f^{0}: M \rightarrow D^{0}$ is a $\mathcal{B}$-preenvelope of $M$ in $R$-Mod, and so we have completed our proof.

By Proposition 3.2, a complex $C$ over any right coherent ring $R$ is Gorenstein flat if and only if each $C^{m}$ is a Gorenstein flat module for $m \in Z$.

Corollary 4.3. Let $R$ be commutative and Noetherian with a dualizing complex. Then every complex of $R$-modules has a Gorenstein flat preenvelope.

Proof. By [5, Theorem 5.7], over such a ring the class of Gorenstein flat modules is closed under direct products. Note also that the class is a Kaplansky class closed under direct limits by [12, Proposition 2.10]. Thus the class of Gorenstein flat modules is preenveloping in $R$-Mod by [12, Theorem 2.5]. By Theorem 4.2, the class of Gorenstein flat complexes is preenveloping in $C(R$-Mod), as desired. 
REMARK 4.4. The result above can also be obtained from Corollary 2.7, Theorem 4.2 and the Theorem on page 2, in [20].

Recall that an $R$-module $M$ is said to be FP-injective (also absolutely pure), if $\operatorname{Ext}_{R}^{1}(A, M)=0$ for all finitely presented $R$-modules $A$. A ring $R$ is left coherent if and only if the class of all FP-injective left $R$-modules is closed under arbitrary direct limits [19, Theorem 3.2]. Thus we have the following result.

COROLlaRY 4.5. Let $R$ be a left coherent ring and $\mathcal{P}$ be the class of all $F P$-injective left $R$-modules. Then every complex of $R$-modules has a dwP-preenvelope.

Proof. Note that the class of FP-injective $R$-modules is preenveloping for any ring $R$ by [10, Proposition 6.2.4]. Thus the result holds by Theorem 4.2.

In the following, we denote by $\mathcal{F}$ the class of flat left $R$-modules. It is showed in [1] and [13] that every complex has a flat cover and a $d w \mathcal{F}$-cover. As mentioned in the introduction that a ring $R$ is right coherent if and only if every complex of $R$-modules has a flat preenvelope, we extend this result to the following.

COROLlary 4.6. A ring $R$ is right coherent if and only if every complex of $R$-modules has a $d w \mathcal{F}$-preenvelope.

Proof. A ring $R$ is right coherent if and only if every $R$-module has a flat preenvelope [10, Proposition 6.5.1], and by Theorem 4.2, if and only if every complex of $R$-modules has a $d w \mathcal{F}$-preenvelope since the class $\mathcal{F}$ is closed under direct limits.

Recall from [14] that a short exact sequence $0 \rightarrow P \rightarrow X \rightarrow X / P \rightarrow 0$ of complexes is pure if for any complex $Y$ of right $R$-modules, the sequence $0 \rightarrow Y \otimes P \rightarrow Y \otimes X \rightarrow Y \otimes X / P \rightarrow 0$ is exact, and a complex is said to be pure injective if it is injective relative to any pure sequence of complexes. According to [14, Proposition 5.1.4], the complex $C^{+}$is pure injective, and the evaluation $C \rightarrow C^{++}$is a pure monomorphism for any complex $C$.

COROLlary 4.7. Let $R$ be commutative and Noetherian with a dualizing complex. Then every pure injective complex $C$ has a Gorenstein injective precover. 
Proof. Since every complex over such a ring has a Gorenstein flat preenvelope by Theorem 4.2, so has $C^{+}$. Let $f: C^{+} \rightarrow G$ be a Gorenstein flat preenvelope of $C^{+}$. Then $G^{+}$is Gorenstein injective by Corollary 3.4. Now it is easily seen that $f^{+}: G^{+} \rightarrow C^{++}$is a Gorenstein injective precover of $C^{++}$ by [9, Corollary 3.2]. On the other hand, $C$ is a direct summand of $C^{++}$by [14, Proposition 5.1.4] since $C$ is pure injective, and then it is easy to see that $\pi f^{+}: G^{+} \rightarrow C$ is a Gorenstein injective precover of $C$, where $\pi: C^{++} \rightarrow C$ is the canonical projection.

Acknowledgements. The work was supported by NSF of China (No. 11101197, 11261050, 11226059) and the Program of Science and Technique of Gansu Province (No. 1107RJZA233). The authors like to thank the referee for valuable suggestions and comments, which improved the present article.

\section{REFERENCES}

[1] S. T. Aldrich - E. E. Enochs - J. R. García Rozas - L. Oyonarte, Covers and envelopes in Groththendieck categories. Flat cover of complexes with applications, J. Algebra, 243 (2001), pp. 615-630.

[2] M. Auslander - I. Reiten, Applications of contravariantly finite subcategories, Adv. Math. 86 (1991), pp. 111-52.

[3] M. Auslander - S. O. Smalø, Preprojective modules over artin algebras, J. Algebra, 66 (1980), pp. 61-122.

[4] H. BASs, Finitistic dimension and a homological characterization of semiprimary rings, Trans. Amer. Math. Soc. 95 (1960), pp. 466-488.

[5] L. W. Christensen - A. Frankild - H. Holm, On Gorenstein projective, injective and flat dimensions-A functor description with applications, J. Algebra, 302 (2006), pp. 231-279.

[6] B. Eckmann - A. Schopf, Ueber injektive moduln. Arch. Math. 4 (2) (1953), pp. $75-78$.

[7] E. E. Enochs, Injective and flat covers, envelopes and resolvents, Israel J. Math. 39 (1981), pp. 33-38.

[8] E. E. Enochs - A. Estrada - A. IAcob, Gorenstein projective and flat complexes over noetherian rings, Math. Nachr. 7 (2012), pp. 834-851.

[9] E. E. ENochs - Z. Y. HuANG, Injective envelopes and (Gorenstein) flat covers, Algebr. Represent. Theor. 15 (2012), pp. 1131-1145.

[10] E. E. EnochS - O. M. G. Jenda, Relative homological algebra, de Gruyter Expositions in Mathematics, Vol. 30, W. de Gruyter, Berlin 2000.

[11] E. E. Enochs - O. M. G. Jenda - J. A. López-Ramos, The existence of Gorenstein flat covers, Math. Scand. 94 (2004), pp. 46-62.

[12] E. E. Enochs - J. A. LóPEZ-Ramos, Kaplansky classes, Rend Sem. Mat. Univ. Padova, 107 (2002), pp. 67-79.

[13] J. GiLlespie, The flat model structure on Ch(R), Tran. Amer. Math. Soc. 356 (8) (2004), pp. 3369-3390. 
[14] J. R. García Rozas, Covers and envelopes in the category of complexes of modules. Boca Raton London New York Washington, D.C. 1999.

[15] R. GöBel - J. TRLIFAJ, Approximations and Endomorphism Algebras of Modules, de Gruyter Expositions in Mathematics, Vol. 41, W. de Gruyter, Berlin-New York 2006.

[16] H. Holm, Gorenstein homological dimensions, J. Pure Appl. Algebra, 189 (2004), pp. 167-193.

[17] Z. K. LIU - C. X. ZhANG, Gorenstein injective complexes of modules over Noetherian rings, J. Algebra, 321 (2009), pp. 1546-1554.

[18] R. B. Warfiel, Purity and algebraic compactness for modules, Pacific J. Math. 28 (1969), pp. 699-719.

[19] B. Stenström, Coherent rings and FP-injective modules, J. London Math. Soc. 2 (2) (1970), pp. 323-329.

[20] J. Stovicek, Deconstructibility and the Hill lemma in Grothendieck categories, Forum Math. 25 (2013), pp. 193-219.

[21] G. YANG - Z. K. LIU, Stability of Gorenstein flat categories, Glasgow Math. J. 54 (1) (2012), pp. 177-191.

Manoscritto pervenuto in redazione l'8 Novembre 2011. 
